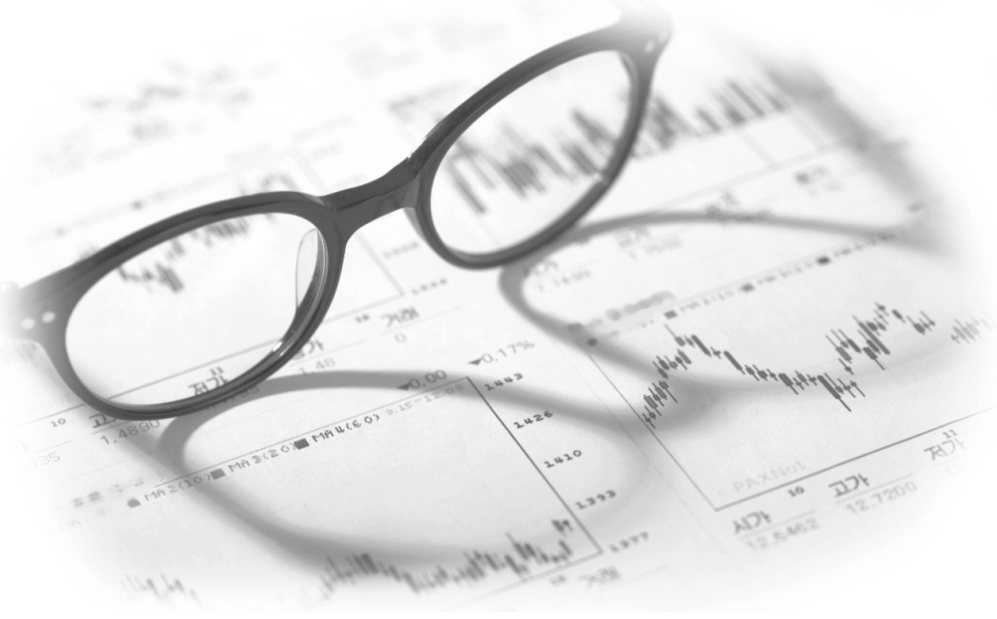

http://dx.doi.org/10.5045/kjh.2012.47.4.241

The Korean Journal of Hematology Volume 47 • Number 4 - December 2012 Perspective

\section{Will JAK1/2 inhibitors change the standard of care for myelofibrosis?}

\author{
Chul Won Jung, M.D., Ph.D. \\ Department of Medicine, Samsung Medical Center, \\ Sungkyunkwan University School of Medicine, Seoul, Korea
}

\section{CURRENT STANDARD OF CARE FOR MYELOFIBROSIS}

Primary myelofibrosis (PMF) is a clonal myeloproliferative neoplasm characterized by conditions such as cytopenia, splenomegaly, megakaryocytic hyperplasia, reactive marrow fibrosis, and other systemic symptoms due to the action of proinflammatory cytokines. The natural course of PMF differs considerably in that high-risk patients show a rapid progression of the disease and a median survival time of 12-24 months. Allogeneic hematopoietic stem cell transplantation (HSCT) has been the only curative treatment so far, but it is associated with a substantial rate of morbidity and mortality frequently related to old age, the presence of severe splenomegaly, and comorbidities. Introduction of reduced-intensity conditioning (RIC) has allowed the geriatric to undergo allogeneic HSCT and has reportedly improved transplant outcome with a progression-free survival of $40-50 \%$ at 3 years.

Nontransplant supportive cares focus on the alleviation of anemia, splenomegaly, and constitutional symptoms. Hydroxyurea is the most commonly used agent for management of splenomegaly, leukocytosis, and thrombocytosis. However, use of other agents such as erythropoiesis-stimulating agents, androgens, anagrelide, interferon, thalido- mide, and lenalidomide in the management of PMF has showed controversial responses in PMF.

\section{JAK1/2 INHIBITORS COULD IMPROVE THE QUALITY OF LIFE}

After the discovery of $J A K 2$ V617F mutation in the cases of $B C R-A B L 1$-negative myeloproliferative neoplasms, hematologists expected that specific Janus kinase 2 (JAK2) inhibitors could change the natural course of myeloproliferative neoplasms, asimatinib has shownin chronic myeloid leukemia. Ruxolitinib (INCB018424) is the first JAK1/2 inhibitor approved in November 2011 by the United States Food and Drug Administration for patients with intermediate- 2 and high-risk PMF. In a randomized phase 3 trial, $42 \%$ of PMF patients in the ruxolitinib group showed $>35 \%$ reduction in spleen volume, whereas the placebo group showed a $0.7 \%$ reduction $(P<0.001)[1]$. The response was durable and was observed not only in patients having the $J A K 2$ V617F mutation but also in patients with wild-type $J A K 2$. Forty-six percent of PMF patients treated with ruxolitinib showed $>50 \%$ improvement of constitutional symptoms, whereas the placebo group showed $5 \%$ improvement. The ruxolitinib group also showed a significant reduction in mortality $(P=0.04)$. Another phase 3 study comparing ruxolitinib with the best supportive care also reported similar findings of marked reduction in spleen volume and improvement in PMF-related symptoms [2]. However, the impact of ruxolitinib on overall survival has not yet been shown. Case-control studies that have compared the effects of ruxolitinib with those of historical controls have reported conflicting survival outcomes $[3,4]$.

Ruxolitinib has been unable to significantly decrease peripheral blood blast count, reverse marrow fibrosis, induce cytogenetic remission, or reduce $J A K 2 \mathrm{~V} 617 \mathrm{~F}$ allele burden. Other limitations of the drug include hematologic toxicities, especially anemia, and the rapid return of splenomegaly

This is an Open Access article distributed under the terms of the Creative Commons Attribution Non-Commercial License (http://creativecommons.org/licenses/by-nc/3.0) which permits unrestricted non-commercial use, distribution, and reproduction in any medium, provided the original work is properly cited. 
and other PMF-related symptoms upon the discontinuation of ruxolitinib. Thus, although ruxolitinib could evidently improve the quality of life by significantly reducing splenomegaly and disease-related symptoms, it is not curative for PMF. Therefore, ruxolitinib may play a definite but limited role in the management of patients with PMF who are unfit for allogeneic HSCT. Several new JAK inhibitors are under clinical development for patients with PMF. However, as JAK2 V617F mutation was not found in one-third of the patients with PMF and because it is not the driver mutation in PMF, development of more potent and specific $J A K 2 \mathrm{~V} 617 \mathrm{~F}$ inhibitors is less likely to change the natural course of PMF. If the two phase III trials show definite survival benefit in ruxolitinib group in long-term follow-up, we have to reappraise its role in the management of PMF patients.

\section{ALLOGENEIC HSCT STILL IS THE ONLY CURATIVE THERAPY FOR MYELOFIBROSIS}

Early reports of allogeneic HSCT demonstrated feasibility and curative potential in PMF patients, even in those with severe splenomegaly. Splenomegaly and marrow fibrosis resolved slowly after transplantation in most of the transplant recipients. Cyclophosphamide with either busulfan or total body irradiation was most commonly used as conditioning regimen, which shows transplant related mortality (TRM) of $30-50 \%$ and long-term survival rate of $30-40 \%$. Introduction of reduced-intensity conditioning decreased TRM such that it became more widely used in the management of patients with PMF. Recent studies reported an increased long-term progression-free survival rate of $40-50 \%$ due to improved TRM; however, this improved TRM was also accompanied by increased relapse of PFM [5]. Fludarabine combined with either intravenous administration of busulfan/melphalan or total body irradiation is the most commonly used RIC regimen. Severe splenomegaly has been associated with poor survival. As JAK inhibitors are not curative in PMF, we suggest that patients with higher-risk PMF whose therapeutic goal is to be cured should proceed to undergo HSCT even when they show a response to JAK1/2 inhibitors. Extensive clinical investigations are required to ascertain whether reduction of splenomegaly and decreasing blood levels of proinflammatory cytokines by using JAK inhibitors during pre-transplant period could improve transplant outcomes.

\section{WILL JAK1/2 INHIBITORS CHANGE THE STANDARD OF CARE FOR MYELOFIBROSIS?}

JAK inhibitors play a definite role in the management of patients with PMF with splenomegaly and other PMF-related symptoms; however, the inhibitors are not curative and do not appear to decrease the risk of leukemic transformation. Allogeneic HSCT is the only curative treatment for PMF, and the advent of RIC regimens has broadened its applications. Future clinical trials should investigate whether use of JAK inhibitors alone to decrease spleen volume and increase performance during the pre-transplant period or use of JAK inhibitors in combination with other active agents would be the better strategy to manage PMF.

\section{REFERENCES}

1. Verstovsek S, Mesa RA, Gotlib J, et al. A double-blind, placebo-controlled trial of ruxolitinib for myelofibrosis. N Engl J Med 2012;366:799-807.

2. Harrison C, Kiladjian JJ, Al-Ali HK, et al. JAK inhibition with ruxolitinib versus best available therapy for myelofibrosis. N Engl J Med 2012;366:787-98.

3. Verstovsek S, Kantarjian HM, Estrov Z, et al. Long-term outcomes of 107 patients with myelofibrosis receiving JAK1/JAK2 inhibitor ruxolitinib: survival advantage in comparison to matched historical controls. Blood 2012;120:1202-9.

4. Tefferi A, Litzow MR, Pardanani A. Long-term outcome of treatment with ruxolitinib in myelofibrosis. N Engl J Med 2011;365:1455-7.

5. Scott BL, Gooley TA, Sorror ML, et al. The Dynamic International Prognostic Scoring System for myelofibrosis predicts outcomes after hematopoietic cell transplantation. Blood 2012;119:265764. 